\title{
THE INFLUENCE OF THE TEMPERATURE, EXTERNAL MAGNETIC FIELD, AND THE THICKNESS OF Pt SUBLAYER ON THE MAGNETOCRYSTALLINE ANISOTROPY CONSTANTS IN Co/Pt MULTILAYERS
}

\author{
L. Louail, A. Azizi, B. Moula and D. Maouche \\ Institute of Physics, University of Setif, (19000) Algeria \\ (Received April 11, 2000; revised version June 13, 2000; \\ in final form September 4, 2000)
}

In this paper we present the behaviour of the magnetocrystalline anisotropy constants of $\left[\mathrm{Co}_{32 \AA}{ }^{\circ} \mathrm{Pt}{ }_{x \AA}\right]_{6}$ multilayers as a function of temperature, external magnetic field, and non-magnetic layer thickness, observed by means of a torque magnetometer in an in-plane configuration. We have determined the magnetocrystalline anisotropy constants from the magnetic torque curves for different temperatures and in different magnetic fields, taking into account the angle difference between the directions of the external field and the magnetization. Among the results of this paper there are the determination of the critical thickness of $\mathrm{Pt}$ and the need to introduce the third order anisotropy constant in the analysis.

PACS numbers: $61.14 . \mathrm{Hg}, 75.30 . \mathrm{Gw}$

\section{Introduction}

In recent years, very interesting phenomena have been shown and received considerable attention in artificially layered magnetic materials. For these fundamental studies as well as for their applications, the magnetism of thin metal films and layered magnetic materials is currently of great interest. The Co/Pt and $\mathrm{Co} / \mathrm{Pd}$ multilayers have been the subject of many recent papers [1-9] because of their wide use in the magnetic recording media [10]. Apart from the potential practical use of these multilayers for high-density computer data storage, the systems are also fascinating because of the effects of quality, composition, and geometrical structure of the interfaces on the anisotropies. The ability to control the anisotropies thus makes these systems ideal for studying the competition between 
field, shape, and magnetic anisotropy effects on the magnetic order in thin film geometries. Among the possible anisotropies, which play a role in the Co/ $\mathrm{Pt}$ system, there are the magnetocrystalline, magnetoelastic, and surface anisotropies.

The study presented in this paper includes the determination of the magnetocrystalline anisotropy constants for different temperatures in magnetic fields ranging from $2 \mathrm{kOe}$ to $10 \mathrm{kOe}$, taking into account the angle difference between the direction of the external magnetic field and the magnetization. The most promising results of this work consist in the need to introduce the third order anisotropy constant in the analysis, which until now has been always neglected and the determination of the critical thickness of $\mathrm{Pt}$.

\section{Samples preparation}

The presence of the magnetic anisotropy is closely related to the anisotropy nature of the artificial multilayer structure itself. The multilayers are prepared by molecular beam epitaxy, after having deposited a buffer layer of monocrystalline $\mathrm{hcp}(0001) \mathrm{Ru}$ of $100 \AA$ thickness on a mica substrate under a pressure below $2 \times 10^{-10}$ mbar. The multilayer consists of six layers of Co with $32 \AA$ thickness each separated by Pt layers of $10 \AA, 15 \AA$, and $20 \AA$ thickness Pt layers. The first Co layer grows directly on the $\mathrm{Ru}$ and the last one is covered with $40 \AA$ of Pt. The multilayer growth is controlled in situ by RHEED as we have described in Ref. [11]. During the growth, the substrate temperature is kept at $240 \mathrm{~K}$ to avoid diffusion at the interface between the Co and the Pt.

The RHEED patterns obtained during the sample growth reveal well-defined structures, which suggest good crystalline quality throughout the deposition. The cobalt grows epitaxially on the $\mathrm{Ru}$ buffer with the hexagonal basal plane parallel to the surface [11]. Using 3D RHEED diffraction analysis on the top layer along the (1010) azimuth, it is convenient to make a distinction between fcc and hcp structures $[12,13]$.

\section{Experimental}

The magnetocrystalline anisotropy is an important quantity dominating the magnetic properties of materials. It is, therefore, essential to know the anisotropy constants so as to determine the properties of materials. In general, the magnetocrystalline anisotropy can be determined by measuring magnetization, magnetic resonance, and magnetic torque curves. Among them, magnetic torque measurements are the most direct and convenient method of determining anisotropy constants. In this technique, the sample is suspended in a field and magnetized to saturation, so that the magnetization lies everywhere in the same easy direction, then the sample will rotate so that this easy direction is aligned with the field. A torque is exerted on the sample, causing it to rotate, and this would be equal to the torque which would be acting on the magnetization vector, causing it to rotate into alignment with the field, if the sample were held clamped. The torque, per unit volume $L$, is given by

$$
L=-\frac{\partial E}{\partial \vartheta}
$$


and therefore the torque on the sample will be $+\partial E / \partial \vartheta$. In practice, the sample is set in a predetermined position and the field is applied. The torsion head is turned until the sample is brought back to its original position and, by separate calibration of the torsion head, this gives a direct reading of the torque exerted. The magnetization measurements were made by means of the superconducting quantum interference device (SQUID) magnetometer.

\section{Results and discussion}

The hysteresis loops of the $\left[\mathrm{Co}_{32 \AA}{ }^{\circ} \mathrm{Pt}{ }_{15 \AA}{ }^{\circ}\right]_{6}$ multilayer, measured by means of the SQUID magnetometer, show that the sample is easy to saturate in the in-plane configuration and that the saturation field is inferior to $5 \mathrm{kOe}$ for the chosen temperature range. For example, at room temperature, a field of $2.5 \mathrm{kOe}$ is sufficient to reach the in-plane multilayer saturation, while at the same temperature a far higher one is necessary $(7.2 \mathrm{kOe})$ to obtain the saturation state in the out-of-plane configuration. When the temperature increases, the sample remains always easy to saturate in the in-plane configuration as shown in Fig. 1.

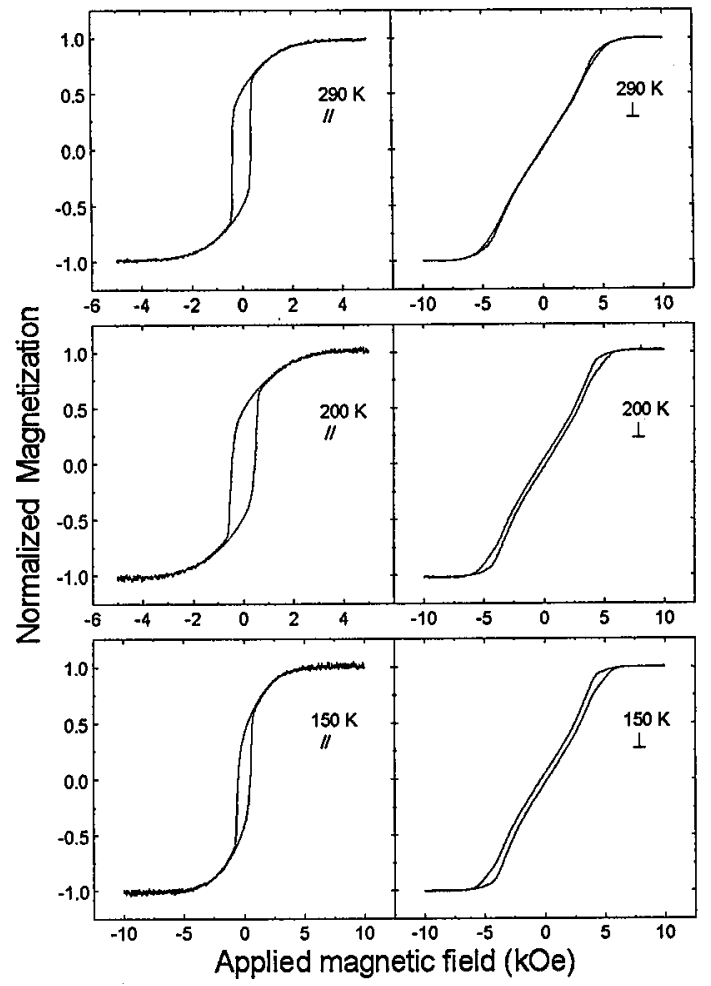

Fig. 1. Hysteresis loops of the $\left[\mathrm{Co}_{32 \AA}{ }^{\circ} \mathrm{Pt}{ }_{15 \AA}\right]_{6}$ multilayer at different temperatures. 
We have analysed the reversible torque curves obtained for $\left[\mathrm{Co}_{32} \AA^{\circ} \mathrm{Pt} \AA_{x}^{\circ}\right]_{6}$ multilayer, at different temperatures and in different external magnetic fields

$$
\Gamma_{\text {rev }}(\theta)=\left[\Gamma^{+}(\theta)+\Gamma^{-}(\theta)\right] / 2,
$$

where $\Gamma^{+}(\theta)$ and $\Gamma^{-}(\theta)$ are the experimental curves obtained from a rotation of the applied magnetic field in the trigonometric direction $(+)$ and in the opposite trigonometric direction $(-)$.

To explain our torque measurements, we have analysed the torque curves using the energy expression of the magnetocrystalline anisotropy

$$
E=K_{u 1} \sin ^{2} \theta+K_{2} \sin ^{4} \theta+K_{3} \sin ^{6} \theta,
$$

where $K_{u 1}, K_{2}$, and $K_{3}$ represent the effective, second, and third order anisotropy constants, respectively. $K_{u 1}=K_{1}-2 \pi M_{\mathrm{s}}^{2}$ includes the magnetocrystalline, the magnetoelastic, and the surface anisotropy terms, $K_{1}$ is the first order anisotropy constant, and $M_{\mathrm{s}}$ is the saturation magnetization of Co $(1420 \mathrm{emu} / \mathrm{cc})$. The interpretation is insufficient if we limit to the second order anisotropy; moreover, the experimental torque curves clearly show the sixth order symmetry.

Thus, the torque per unit volume, $L$, resulting from this anisotropy energy can be expressed as

$$
L=\left(K_{u 1}+K_{2}+\frac{15}{16} K_{3}\right) \sin 2 \theta-0.5\left(K_{2}+1.5 K_{3}\right) \sin 4 \theta+\frac{3}{16} K_{3} \sin 6 \theta,
$$

where

$$
\theta=\theta^{\prime}-\sin ^{-1}\left(-L / M_{\mathrm{s}} H\right)
$$

$\theta^{\prime}$ being the angle between the $c$ axis and the external magnetic field direction, and $\theta$ - the angle between the magnetization direction and that of the $c$ axis. We have introduced, in Eq. (3), the correction of angle difference between the directions of the external magnetic field and the magnetization.

By fitting the experimental torque curves with expression (4) we deduce the behaviour of the anisotropy constants versus temperature, external magnetic field, and Pt layer's thickness.

In an external magnetic field of $5 \mathrm{kOe}$, the effective anisotropy constant $K_{u 1}$ slightly decreases with increasing temperature and this happens for all thicknesses of the Pt non-magnetic layer, as shown in Fig. 2. When the external magnetic field becomes strong $(10 \mathrm{kOe}), K_{u 1}$ takes its usual behaviour; it strongly decreases with increasing temperature as shown in Fig. 3. This is a usual behaviour, since for hcp Co, $K_{u 1}$ rapidly decreases with increasing temperature. Such behaviour, predicted by Zener's theory [14] using either the localized electron [15, 16] or the itinerant electron [16] models, was confirmed and explained by Carr's introduction of thermal expansion effect of the lattice parameter ratio $c / a$ [15]. The decrease in $K_{u 1}$ with temperature is also explained by the calculation of Mori et al. [17] based on the Fermi distribution effect, in which this decrease can be expressed with terms proportional to $T^{2}$ and $T^{5 / 2}$. Furthermore, the importance of $K_{u 1}$ behaviour was clearly shown by Carr [15], and the dependence of the lattice parameter ratio $c / a$ was shown by Muller et al. [18] and White [19].

Also we have determined the anisotropy values for $K_{2}$ and $K_{3}$, we have remarked that the $K_{3}$ values, reported in the Table for an external field of $10 \mathrm{kOe}$, 


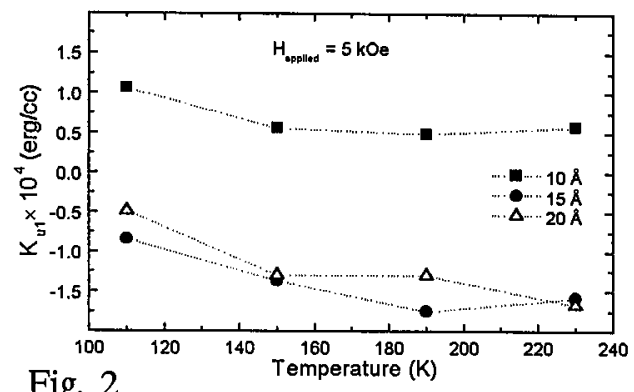

Fig. 2

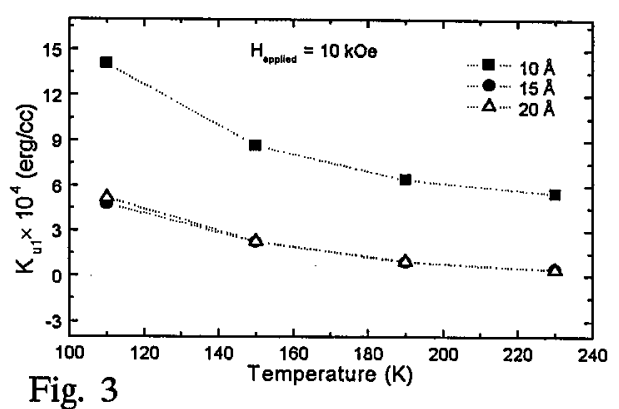

Fig. 3

Fig. 2. $K_{u 1}$ at different temperatures for multilayers of $10 \AA, 15 \AA$, and $20 \AA$ of Pt thickness in an external magnetic field of $5 \mathrm{kOe}$.

Fig. 3. $K_{u 1}$ at different temperatures for multilayers of $10 \AA, 15 \AA$, and $20 \AA$ of Pt thickness in an external magnetic field of $10 \mathrm{kOe}$.

\section{TABLE}

Magnetic anisotropy values of second and third order anisotropy constants of $\left[\mathrm{Co}_{32 \AA} \mathrm{Pt}{ }_{x \AA}^{\circ}\right]_{6}$ in an applied magnetic field of $10 \mathrm{kOe}$, at different temperatures. $K_{3}$ cannot be neglected in comparison with $K_{2}$.

\begin{tabular}{cc|c|c|c|c}
\hline \hline \multicolumn{2}{c|}{ Temperature $[\mathrm{K}]$} & 110 & 150 & 190 & 230 \\
\hline $\operatorname{Pt}(10 \AA)$ & $K_{2}\left(\times 10^{4} \mathrm{erg} / \mathrm{cc}\right)$ & 3.70 & 3.32 & 3.42 & 3.28 \\
& $K_{3}\left(\times 10^{4} \mathrm{erg} / \mathrm{cc}\right)$ & 2.65 & 2.35 & 2.40 & 2.25 \\
\hline $\operatorname{Pt}(15 \AA)$ & $K_{2}\left(\times 10^{4} \mathrm{erg} / \mathrm{cc}\right)$ & 5.72 & 5.00 & 4.68 & 4.22 \\
& $K_{3}\left(\times 10^{4} \mathrm{erg} / \mathrm{cc}\right)$ & 3.75 & 3.25 & 3.15 & 2.85 \\
\hline $\operatorname{Pt}(20 \AA)$ & $K_{2}\left(\times 10^{4} \mathrm{erg} / \mathrm{cc}\right)$ & 5.16 & 4.26 & 3.86 & 3.50 \\
& $K_{3}\left(\times 10^{4} \mathrm{erg} / \mathrm{cc}\right)$ & 3.35 & 2.75 & 2.50 & 2.25
\end{tabular}

are much more important compared to $K_{u 1}$ and $K_{2}$ and then $K_{3}$ cannot be neglected as we did for the perpendicular configuration [11,20,21] and as until now it has been always practiced.

The variation of anisotropy constants with the magnetic field in the ferromagnetic metals is one of the important properties in the magnetic character of these materials. Ono and Yamada [22] obtained a very small variation of $K_{u 1}$ with the external magnetic field; in contrast to other results as the ones obtained by Chikazumi et al. [23] in which $K_{u 1}$ considerably increases with an increasing external magnetic field at very low temperature. Chikazumi et al. have found an increase in $K_{u 1}$ from $0.5 \times 10^{6} \mathrm{erg} / \mathrm{cc}$ in an external field of $5 \mathrm{kOe}$ up to $3.2 \times 10^{6} \mathrm{erg} / \mathrm{cc}$ in $10 \mathrm{kOe}$ at $4.2 \mathrm{~K}$. According to the study of Ono and Yamada [22], this wide variation of $K_{u 1}$ with the external magnetic field, seems to be due to the analysing method in which the variation effect of the difference between the magnetization and the external magnetic field directions was not properly taken into consideration. 


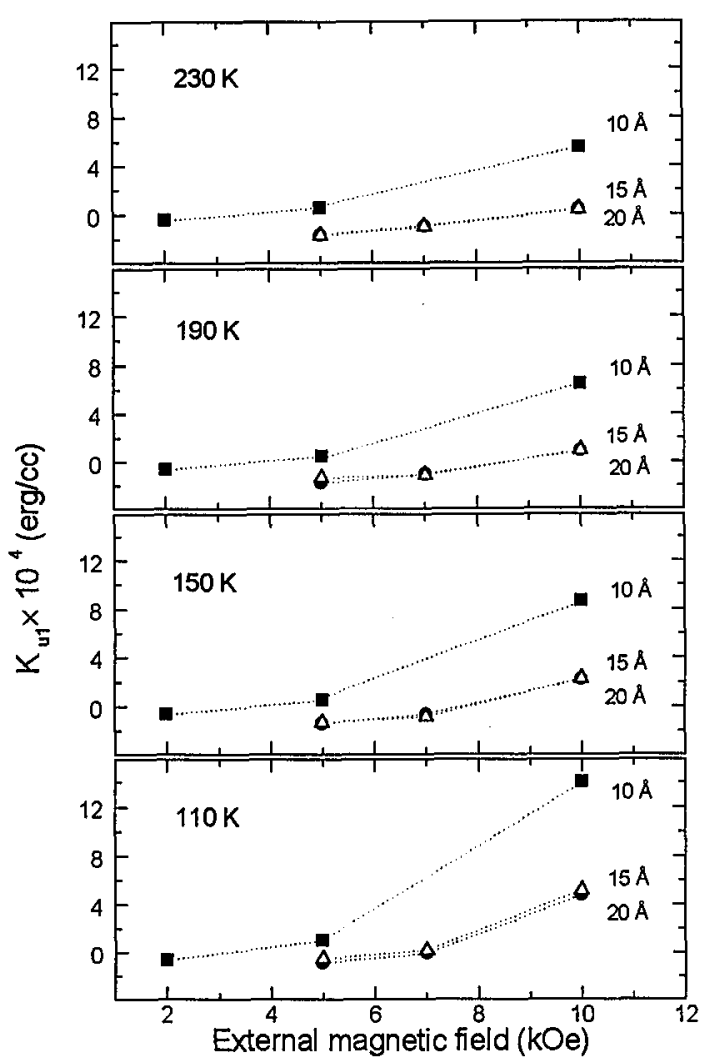

Fig. 4. The influence of external magnetic field on $K_{u 1}$ at different temperatures.

In our multilayers, when the applied magnetic field is weak, $K_{u 1}$ does not practically change with the applied magnetic field. But in a strong applied field, $K_{u 1}$ becomes sensitive to the magnetic field; it clearly increases with increasing the applied field. This increase is more and more important at lower temperatures as shown in Fig. 4. But this variation cannot be explained as Ono and Yamada did in Ref. [22] because, on the one hand, in our analysis we have taken into consideration the difference between the direction of the magnetisation and the external magnetic field; on the other hand, this dependence is clearly seen in the experimental curves of the magnetic torque as shown in Fig. 5 , an example taken for $15 \AA$ of $\mathrm{Pt}$ at $110 \mathrm{~K}$. This variation of the magnetic torque curves with the external magnetic field at any temperature is similar to that found by Wu et al. [24] who based their work on the Stoner-Wohlfarth model [25, 26], by also taking into account the difference between the directions of the magnetisation and the external field.

We may possibly find an explanation in the theory of Usami and Moriya [27], in which the effect of longitudinal fluctuation of spins plays a role in the variation 


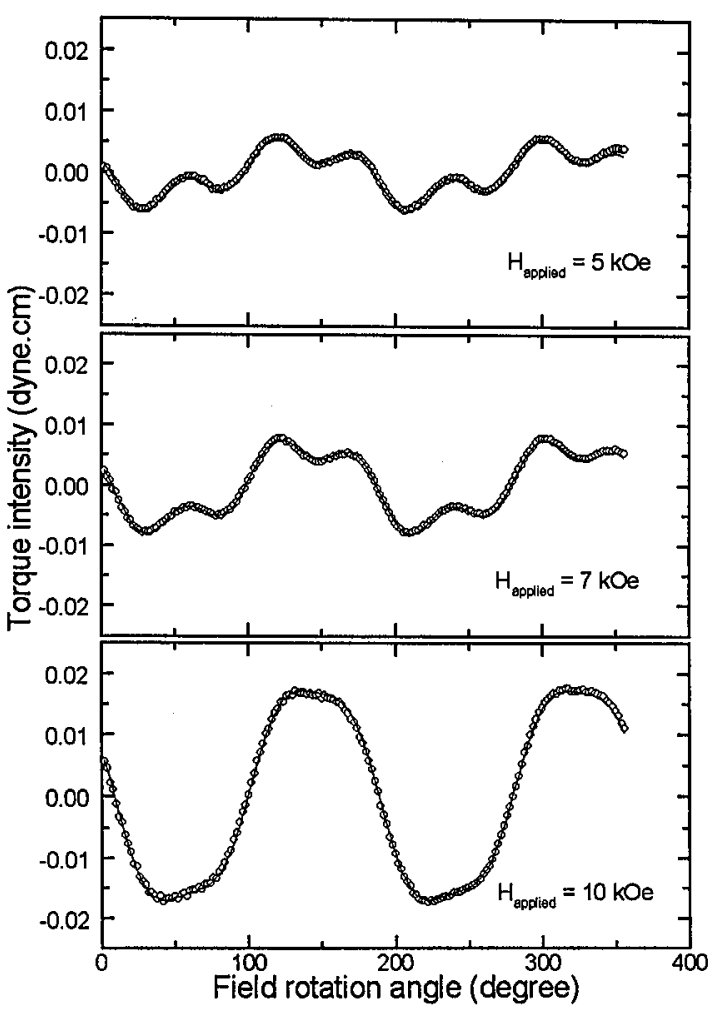

Fig. 5. Experimental curves of the magnetic torque of $\left[\mathrm{Co}_{32 \AA} \mathrm{Pt}_{15 \AA}\right]_{6}$ at $110 \mathrm{~K}$ in different applied magnetic fields. The measured data appear as open circles and the fit by line.

of $K_{u 1}$ because this effect can change the temperature of the local magnetic moment [28]. In fact, from the effective anisotropy constant which may approximately be expressed in three terms [29]: the magnetization dependent term $K_{\mathrm{M}}(T, H)$, the lattice parameter dependent term $K_{\mathrm{L}}(T)$, and the Fermi level-dependent term $K_{\mathbf{F}}(T, H)$

$$
K_{u 1}(T, H)=K_{u 1}(0,0)+K_{\mathrm{M}}(T, H)+K_{\mathrm{L}}(T)+K_{\mathrm{F}}(T, H),
$$

we think that the change of $K_{u 1}$ at low temperature is essentially caused by the term depending on the lattice parameters $K_{\mathrm{L}}(T)$ and therefore by the change in the lattice parameter ratio $c / a$. Moreover, and as it was shown in Refs. [30,31], the contribution of the magnetostriction energy to the effective anisotropy constant is also important and may play a significant role here; the previous papers [32-34] suggest that small strains of order of $0.01 \%$ can lead to anisotropy with magnitudes comparable to those observed in the present samples.

The dependence of $K_{u 1}$ for samples with different non-magnetic layer thicknesses as a function of temperature is shown in Figs. 2 and $3, K_{u 1}$ strongly decreases with increasing thickness Pt up to $15 \AA$. We can consider that the thickness of $15 \AA$ of $\mathrm{Pt}$ represents the critical thickness in the $K_{u 1}$ variation; we think that 
this critical thickness of the non-magnetic layer represents a limit so that the Co layers react between them, beyond the value of $15 \AA$, the Co layers move away and no change of $K_{u 1}$ has been seen. This is possible due to the fact that the residual strain decreases continuously with increasing the Pt thickness because the interface plays a major role due to the reduced co-ordination symmetry [35], the altered electronic structure [36], or the strain and interface dislocations [37].

\section{Conclusion}

In this work, we have determined the magnetocrystalline anisotropy constants of the $\left[\mathrm{Co}_{32 \AA}{ }^{\circ} \mathrm{Pt}{ }_{x \AA}^{\circ}\right]_{6}$ multilayer as a function of temperature, external magnetic field and non-magnetic layer thickness. The behaviour of $K_{u 1}$ is usual; it decreases with increasing temperature especially at a strong field; we think that this is caused by the anisotropy term dependent on the change of lattice parameter ratio $c / a$. The variation of $K_{u 1}$ with the external magnetic field at lower temperatures is in agreement with other author results. The promising result of this work consists in the need to introduce the third order anisotropy constant in the analysis. Also we have determined a critical value of non-magnetic layer thickness (15 $\AA$ ), beyond this value, $K_{u 1}$ does change neither in form nor in values.

\section{Acknowledgments}

This work has been realised at the Institut de Physique et de Chimie des Matériaux de Strasbourg (France) in the Groupe d'Etude des Matériaux Métalliques. We would like to thank Dr K. Ounadjela and Prof. H. Danan for stimulating discussions.

\section{References}

[1] B. Heinrich, J.F. Cochran, Adv. Phys. 42, 523 (1994) and references therein.

[2] P.F. Carcia, A.D. Meinhaldt, A. Suna, Appl. Phys. Lett. 47, 178 (1985).

[3] W.B. Zeper, F.J. Greidanus, P.F Carcia, C.R. Fincher, J. Appl. Phys. 65, 4971 (1989).

[4] F.J.A den Broeder, H.C. Donkersloot, H.J.G. Draaisma, W.J.M. de Jonge, J. Appl. Phys. 61, 4317 (1987).

[5] C. Chappert, K. Le Dang, P. Beauvillain, H. Hurdequint, D. Renard, Phys. Rev. $B$ 34, 3192 (1986).

[6] F.J.A. den Broeder, W. Hoving, P.J.H. Bloemen, J. Magn. Magn. Mater. 93, 562 (1991).

[7] P.F. Carcia, J. Appl. Phys. 63, 5066 (1988).

[8] F.J.A. den Broeder, D. Kuiper, H.C. Donkersloot, W. Hoving, Appl. Phys. A 49, 507 (1989).

[9] P.F. Carcia, Z.G. Li, M. Reilly, W.B. Zeper, J. Appl. Phys. 73, 6424 (1993).

[10] M. Hartmann, B.A.J. Jacobs, J.J.M. Braat, Philips Tech. Rev. 42, 37 (1985). 
[11] L. Louail, K. Ounadjela, R.L. Stamps; J. Magn. Magn. Mater. 167, L189 (1997).

[12] K. Ounadjela, P. Vennegues, Y. Henry, A. Michel, V. Pierron, J. Arabski, Phys. Rev. B 49, 8561 (1994).

[13] Y. Henry, V. Pierron, P. Vennegues, K. Ounadjela, J. Appl. Phys. 76, 2817 (1994). 